\title{
Generalized Landau-de Gennes theory of uniaxial and biaxial nematic liquid crystals
}

\author{
M. C. J. M. Vissenberg and S. Stallinga* \\ Philips Research Laboratories, Professor Holstlaan 4, 5656 AA Eindhoven, The Netherlands \\ G. Vertogen \\ Institute for Theoretical Physics, University of Nijmegen, Toernooiveld, 6525 ED Nijmegen, The Netherlands
}

(Received 25 September 1996)

\begin{abstract}
The generalized Landau-de Gennes theory for uniaxial and biaxial nematic liquid crystals is analyzed using tensor analysis. In this way all terms allowed by symmetry appear in the free-energy expression. This improves the quantitative shortcomings of the commonly used theory. It appears that uniaxial nematic liquid crystals are described by 12 independent generalized elastic terms and that biaxial nematic liquid crystals are described by 48 independent generalized elastic terms. For practical purposes, several approximations based on symmetry arguments are discussed. The theory is applied to describe the variation in order and orientation near a surface. The relevance of the theory to the debate on the surface elastic constant $K_{13}$ is pointed out. [S1063-651X(97)06004-2]
\end{abstract}

PACS number(s): 61.30.-v, 62.20.Dc, 64.70.Md

\section{INTRODUCTION}

More than two decades ago, de Gennes applied the Landau theory of second-order phase transitions $[1,2]$ to the firstorder isotropic-uniaxial nematic phase transition [3]. This Landau-de Gennes theory proved to be useful for the description of other phase transitions as well, e.g., the isotropic-biaxial nematic transition and the uniaxial nematic-biaxial nematic transition [4,5]. The influence of spatial variations of the local order parameter is taken into account in the generalized Landau-de Gennes theory (GLGT) [6-9]. This GLGT naturally combines Landau theory with orientational elasticity theory, as the local order of a nematic liquid crystal is determined by an amplitude (which describes the "degree" of order) and an orientation. Therefore, the GLGT can be used to describe phenomena which cannot be satisfactorily described by the original Landau-de Gennes theory and elasticity theory separately. Such phenomena are expected when both the degree of order and the orientation are important, as is the case with, e.g., phase transitions in confined geometries and wetting phenomena [10-14], surface-induced bulk alignment [15-17], and disclinations $[18,19]$.

In contrast to its wide application, the GLGT is clearly limited from a quantitative point of view, as the GLGT expression for the free-energy density of orientational deformations is not identical to the ones that are well known for either uniaxial [20-24] or biaxial [25-30] nematic liquid crystals. For example, according to the GLGT known from the literature [3,6-9], the Frank elastic constants [22] for splay, twist, and bend $K_{11}, K_{22}$, and $K_{33}$, respectively, satisfy the relations

\footnotetext{
*Author to whom correspondence should be addressed. Electronic address: stalling@natlab.research.philips.com
}

$$
\begin{gathered}
K_{11}=K_{33}>K_{22}, \\
K_{i i} \propto Q_{1}^{2},
\end{gathered}
$$

where $Q_{1}$ is the scalar order parameter. In general these relations do not hold: the degeneracy of $K_{11}$ and $K_{33}$ is removed when the Landau-de Gennes expansion is extended to third order in $Q_{1}$ [31]. Apparently, the presently known GLGT is an approximation of a more complete theory. The aim of this paper is to investigate this complete theory. Our analysis is based on symmetry principles only. In this way, all effects that are allowed by symmetry are incorporated in the theory. Moreover, the physical assumptions underlying the commonly used approximate GLGT become quite clear in the present analysis.

This paper is organized as follows. For the sake of clarity the main concepts are reviewed in Sec. II. The symmetries of the different nematic phases and the corresponding tensor order parameters are discussed and the general expressions for the Landau free-energy density and the elastic freeenergy density are given. Special attention is paid to the distinction between the surface free-energy density and the bulk free-energy density. In Sec. III the GLGT of uniaxial and biaxial nematic liquid crystals is presented. We derive the most general free-energy expression that is allowed by symmetry and discuss various approximations based on approximate symmetries. The GLGT known from the literature is obtained when the symmetry is approximately isotropic. In Sec. IV the theory is applied to the coupled variation of the order parameters and the orientation close to a surface that favors an ordering of the nematic liquid crystal different from the thermodynamically stable ordering. The result is directly related to the debate on the surface elastic constant $K_{13}$. The paper is concluded in Sec. V by a short summary of the main results.

\section{THEORY}

\section{A. The tensor order parameter}

The macroscopic tensor order parameter $\mathbf{Q}$ is defined as the anisotropic part of a susceptibility $\mathbf{T}[6-8]$, 


$$
Q_{i j}=G\left(T_{i j}-\frac{1}{3} \operatorname{Tr}(\mathbf{T}) \delta_{i j}\right),
$$

where $\mathbf{T}$ may represent the magnetic susceptibility tensor $\boldsymbol{\chi}$ or the dynamic dielectric tensor $\boldsymbol{\epsilon}(\omega)$ at a standard frequency $\omega$. The factor $G$ is an arbitrary normalization constant. For convenience, we choose $G>0$ and having dimensions such that $\mathbf{Q}$ becomes dimensionless.

The tensor order parameter $\mathbf{Q}$ being real, symmetric, and of zero trace, can be written in the following diagonal form in a suitably chosen local frame of orthogonal eigenvectors $(\boldsymbol{l}(\boldsymbol{r}), \boldsymbol{m}(\boldsymbol{r}), \boldsymbol{n}(\boldsymbol{r}))$ :

$$
\boldsymbol{Q}=\left(\begin{array}{ccc}
-\frac{1}{3}\left(Q_{1}-Q_{2}\right) & 0 & 0 \\
0 & -\frac{1}{3}\left(Q_{1}+Q_{2}\right) & 0 \\
0 & 0 & \frac{2}{3} Q_{1}
\end{array}\right) .
$$

The representation of the tensor order parameter in the space-fixed frame $\left(\boldsymbol{e}_{x}, \boldsymbol{e}_{y}, \boldsymbol{e}_{z}\right)$ reads

$$
Q_{\alpha \beta}=Q_{1}\left(N_{\alpha \beta}-\frac{1}{3} \delta_{\alpha \beta}\right)+\frac{1}{3} Q_{2}\left(L_{\alpha \beta}-M_{\alpha \beta}\right),
$$

where we have used the definitions

$$
\begin{gathered}
L_{\alpha \beta}=l_{\alpha} l_{\beta}, \\
M_{\alpha \beta}=m_{\alpha} m_{\beta}, \\
N_{\alpha \beta}=n_{\alpha} n_{\beta}
\end{gathered}
$$

and the completeness of the set of eigenvectors

$$
L_{\alpha \beta}+M_{\alpha \beta}+N_{\alpha \beta}=\delta_{\alpha \beta} .
$$

The nematic phase with the highest symmetry is the uniaxial nematic phase $N_{u}$, which has a continuous rotational symmetry around a unique axis. This symmetry axis is thought to coincide with the eigenvector $\boldsymbol{n}$. The two eigenvectors $\boldsymbol{l}$ and $\boldsymbol{m}$ are degenerate. The order of the $N_{u}$ phase with respect to the isotropic phase is described by the uniaxial tensor order parameter

$$
Q_{\alpha \beta}^{u}=Q_{1}\left(N_{\alpha \beta}-\frac{1}{3} \delta_{\alpha \beta}\right) .
$$

The $N_{u}$ phase is nonchiral, i.e., symmetric under spatial inversion, and nonpolar, i.e., the states described by $\boldsymbol{n}$ and $-\boldsymbol{n}$ are indistinguishable. The chiral variant of this phase is the cholesteric or chiral nematic $N_{u}^{*}$ phase.

A nematic phase with lower symmetry is the biaxial nematic phase $N_{b}$. Here the continuous rotational symmetry around $\boldsymbol{n}$ is also broken: the two eigenvectors $\boldsymbol{l}$ and $\boldsymbol{m}$ are no longer degenerate. The order of the $N_{b}$ phase with respect to the isotropic phase is described by the full tensor order parameter (3). The chiral variant of this phase is denoted as $N_{b}^{*}$.

The $N_{b}$ phase is more ordered than the $N_{u}$ phase. The order of the $N_{b}$ phase with respect to the $N_{u}$ phase is described by the biaxial tensor order parameter $\mathbf{Q}^{b}$, which is obtained by subtracting both the isotropic and the uniaxial part of the tensor $\boldsymbol{T}$,

$$
Q_{\alpha \beta}^{b}=\frac{1}{3} Q_{2}\left(L_{\alpha \beta}-M_{\alpha \beta}\right) .
$$

Recently, a tensor order parameter with nonzero trace has been used by Tolédano et al. [32] in order to describe the phase diagrams of lyotropic nematic and lyotropic cholesteric systems. The additional scalar order parameter that is thus introduced describes a change in the shape of the micelles that constitute the lyotropic system.

The properties of polar liquid crystals have been systematically descibed by Longa and Trebin [33] using the traceless, symmetric tensor order parameter $Q_{\alpha \beta}$ together with a polar field $P_{\alpha}$. When $P_{\alpha}=0$, the GLGT expression known from the literature is obtained.

In the following, we confine ourselves to nonpolar, thermotropic nematic and cholesteric liquid crystals with uniaxial and biaxial symmetries. The uniaxial systems are descibed by the uniaxial tensor order parameter $\mathbf{Q}^{u}$. The biaxial systems can be descibed by the total tensor order parameter $\mathbf{Q}$, but sometimes a description in terms of two separate tensor order parameters $\mathbf{Q}^{u}$ and $\mathbf{Q}^{b}$ is more appropriate. This distinction between a separate uniaxial and biaxial tensor order parameter, which, to our knowledge, has not been made before, is of importance when discussing the different approximations to the GLGT presented in Sec. III. When the distinction is not made and only the total tensor order parameter $\mathbf{Q}$ is used, the GLGT known from the literature is obtained.

\section{B. The Landau free-energy density}

Consider an arbitrary thermodynamic system that is characterized by a uniform temperature $T$, a uniform pressure $p$, and some tensor order parameter $O_{\alpha \beta}(\boldsymbol{r})$. Such a system can be described by the so-called Landau free-energy density $g_{L}$, which is a function of the temperature $T$, the pressure $p$, the local order parameter $O_{\alpha \beta}(\boldsymbol{r})$, and its spatial derivatives of all orders, denoted as $\partial_{\alpha}^{(n)} O_{\beta \gamma}(\boldsymbol{r})$. For sufficiently smooth variations of the order parameter, the Landau freeenergy density can be expanded in powers of the spatial derivatives of the order parameter. Usually only terms that are linear in the first-order derivatives, terms that are quadratic in the first-order derivatives, and terms that are linear in the second-order derivatives are taken into account,

$$
\begin{aligned}
g_{L}\left(\boldsymbol{O}(\boldsymbol{r}), \boldsymbol{\partial}^{(n)} \boldsymbol{O}(\boldsymbol{r}), T, p\right) & =g_{L, u}(\boldsymbol{O}(\boldsymbol{r}), T, p)+k_{\alpha \beta \gamma}(\boldsymbol{O}(\boldsymbol{r}), T, p) \partial_{\alpha} O_{\beta \gamma}(\boldsymbol{r}) \\
& +K_{\alpha \beta \gamma \lambda \mu \nu}(\boldsymbol{O}(\boldsymbol{r}), T, p) \partial_{\alpha} O_{\beta \gamma}(\boldsymbol{r}) \partial_{\lambda} O_{\mu \nu}(\boldsymbol{r}) \\
& +\mathcal{K}_{\alpha \beta \gamma \delta}(\boldsymbol{O}(\boldsymbol{r}), T, p) \partial_{\alpha} \partial_{\beta} O_{\gamma \delta}(\boldsymbol{r})+\cdots,
\end{aligned}
$$

where $g_{L, u}(\boldsymbol{O}(\boldsymbol{r}), T, p)$ denotes the Landau free-energy density of the system when the order parameter is uniform. The other terms in the expansion describe the elastic free-energy density. The tensors $\boldsymbol{k}, \boldsymbol{K}, \mathcal{K}, \ldots$ have the symmetry of the lower symmetric $\left(O_{\alpha \beta} \neq 0\right)$ phase. They are functions of the local order parameter $O_{\alpha \beta}(\boldsymbol{r})$, the temperature $T$, and pressure $p$.

The Landau free-energy density $g_{L}$ can also be expanded in powers of $O_{\alpha \beta}$. The expansion of $g_{L, u}$ with respect to the $O_{\alpha \beta}=0$ phase reads 


$$
\begin{aligned}
& g_{L, u}(\boldsymbol{O}(\boldsymbol{r}), T, p) \\
&= g_{0}(T, p)+Z_{\alpha \beta} O_{\alpha \beta}+\frac{1}{2} A_{\alpha \beta \gamma \delta} O_{\alpha \beta} O_{\gamma \delta} \\
&-\frac{1}{3} B_{\alpha \beta \gamma \delta \epsilon \zeta} O_{\alpha \beta} O_{\gamma \delta} O_{\epsilon \zeta} \\
&+\frac{1}{4} C_{\alpha \beta \gamma \delta \epsilon \zeta \eta \theta} O_{\alpha \beta} O_{\gamma \delta} O_{\epsilon \zeta} O_{\eta \theta}+\cdots,
\end{aligned}
$$

where $g_{0}$ denotes the Gibbs free-energy density of the higher symmetric $O_{\alpha \beta}=0$ phase. The tensors $\boldsymbol{k}, \boldsymbol{K}$, and $\mathcal{K}$ can be expanded as well:

$$
\begin{gathered}
k_{\alpha \beta \gamma}(\boldsymbol{O}(\boldsymbol{r}), T, p)=k_{\alpha \beta \gamma}^{0}(T, p)+\cdots, \\
K_{\alpha \beta \gamma \lambda \mu \nu}(\boldsymbol{O}(\boldsymbol{r}), T, p)=K_{\alpha \beta \gamma \lambda \mu \nu}^{0}(T, p)+\cdots, \\
\mathcal{K}_{\alpha \beta \gamma \delta}(\boldsymbol{O}(\boldsymbol{r}), T, p)=\mathcal{K}_{\alpha \beta \gamma \delta}^{0}(T, p)+\cdots
\end{gathered}
$$

It is important to note that the tensors $\mathbf{Z}, \mathbf{A}, \mathbf{B}, \mathbf{C}, \mathbf{k}^{0}, \mathbf{K}^{0}$, $\mathcal{K}^{0}, \ldots$ have the symmetry of the higher symmetric $\left(O_{\alpha \beta}=0\right)$ phase. They are functions of the temperature $T$ and pressure $p$.

\section{Surface terms}

A number of terms appearing in expansion (8) of the Landau free-energy density can be written in the form of divergences. These terms are called "surface terms," as they only contribute to the surface free energy according to Gauss's theorem. The remaining terms in expansion (8) are referred to as "bulk terms." In the thermodynamic limit the contribution of the surface terms to the total free energy is negligible compared to the contribution of the bulk terms. In this section, we derive the general form of the surface terms that occur in expansion (8).

By definition, the general form of a surface term is

$$
\partial_{\alpha} S_{\alpha}\left(\boldsymbol{O}, \boldsymbol{\partial}^{(n)} \boldsymbol{O}\right),
$$

where $S$ can be any vector function of the order parameter and its spatial derivatives. Expanding this function in powers of the spatial derivatives,

$$
S_{\alpha}\left(\boldsymbol{O}, \boldsymbol{\partial}^{(n)} \boldsymbol{O}\right)=S_{\alpha}^{0}(\boldsymbol{O})+S_{\alpha \beta \gamma \delta}^{1}(\boldsymbol{O}) \partial_{\beta} O_{\gamma \delta}+\cdots,
$$

one finds that the two lowest-order terms comprise all the surface terms in Eq. (8):

$$
\begin{gathered}
\partial_{\alpha} S_{\alpha}^{0}=\frac{\partial S_{\alpha}^{0}}{\partial O_{\beta \gamma}} \partial_{\alpha} O_{\beta \gamma}, \\
\partial_{\alpha}\left(S_{\alpha \beta \gamma \delta}^{1} \partial_{\beta} O_{\gamma \delta}\right)=\frac{\partial S_{\alpha \beta \gamma \delta}^{1}}{\partial O_{\epsilon \zeta}} \partial_{\alpha} O_{\epsilon \zeta} \partial_{\beta} O_{\gamma \delta}+S_{\alpha \beta \gamma \delta}^{1} \partial_{\alpha} \partial_{\beta} O_{\gamma \delta} .
\end{gathered}
$$

We note that surface terms of the form (13a) are absent in the uniaxial and biaxial nematic phases, due to the nonpolar character of these phases. For surface terms of the form (13b), we make a distinction between symmetric and antisymmetric surface terms, as in Ref. [30]. For the antisymmetric surface terms (with $S_{\beta \alpha \gamma \delta}^{1}=-S_{\alpha \beta \gamma \delta}^{1}$ ), the terms that are linear in the second-order derivative vanish. On the other hand, there is a one-to-one correspondence between symmetric surface terms (with $S_{\beta \alpha \gamma \delta}^{1}=S_{\alpha \beta \gamma \delta}^{1}$ ) and terms that are linear in the second-order derivative.

\section{THE GLGT OF NEMATIC LIQUID CRYSTALS}

\section{A. Uniform systems: Phase transitions}

The $I-N_{u}$ phase transition may be described by an expansion of $g_{L, u}$ in powers of the uniaxial tensor order parameter $\mathbf{Q}^{u}$. The tensors that appear in this expansion must have the symmetry of the isotropic $\left(\mathbf{Q}^{u}=\mathbf{0}\right)$ phase, i.e., these tensors are combinations of scalars $s$, Kronecker deltas $\delta_{\alpha \beta}$, and products $p \varepsilon_{\alpha \beta \gamma}$ of a pseudoscalar $p$ with the Levi-Civita symbol $\varepsilon_{\alpha \beta \gamma}$ (which is a pseudotensor).

The tensor $p \varepsilon_{\alpha \beta \gamma}$ does not appear in the expansion of $g_{L, u}$, as all tensors in this expansion must have an even number of indices [see Eq. (9)]. In fact, the general expansion requires only the two lowest-order contractions of the tensor order parameter $[4,8,33]$,

$$
\begin{gathered}
Q_{\alpha \beta}^{u} Q_{\beta \alpha}^{u}=\frac{2}{3} Q_{1}^{2}, \\
Q_{\alpha \beta}^{u} Q_{\beta \gamma}^{u} Q_{\gamma \alpha}^{u}=\frac{2}{9} Q_{1}^{3},
\end{gathered}
$$

which gives rise to the well-known expression for the Landau expansion near the $I-N_{u}$ transition,

$$
g_{L, u}=g_{\text {iso }}+\frac{1}{3} A Q_{1}^{2}-\frac{2}{27} B Q_{1}^{3}+\frac{1}{9} C Q_{1}^{4}+\cdots,
$$

where the expansion up to $Q_{1}^{4}$ suffices to describe the experimentally observed first-order phase transition. The coefficients $A, B, C, \ldots$ and the free-energy density of the isotropic phase $g_{\text {iso }}$ are smooth functions of $T$ and $p$. A discussion of the $I-N_{u}$ transition and the Landau expansion (15) can be found in, e.g., Refs. [6-9].

The $I-N_{b}$ phase transition is described by an expansion of $g_{L, u}$ in powers of the full tensor order parameter Q. All tensors that appear in this expansion must have the symmetry of the isotropic $(\mathbf{Q}=\mathbf{0})$ phase. The general expansion requires only the two lowest-order contractions of the full tensor order parameter [8],

$$
\begin{gathered}
Q_{\alpha \beta} Q_{\beta \alpha}=\frac{2}{3}\left(Q_{1}^{2}+\frac{1}{3} Q_{2}^{2}\right), \\
Q_{\alpha \beta} Q_{\beta \gamma} Q_{\gamma \alpha}=\frac{2}{9} Q_{1}\left(Q_{1}^{2}-Q_{2}^{2}\right),
\end{gathered}
$$

yielding

$$
\begin{aligned}
g_{L, u}= & g_{\text {iso }}+\frac{1}{3} A\left(Q_{1}^{2}+\frac{1}{3} Q_{2}^{2}\right)-\frac{2}{27} B Q_{1}\left(Q_{1}^{2}-Q_{2}^{2}\right) \\
& +\frac{1}{9} C\left(Q_{1}^{2}+\frac{1}{3} Q_{2}^{2}\right)^{2}+\frac{4}{135} D\left(Q_{1}^{2}+\frac{1}{3} Q_{2}^{2}\right) Q_{1}\left(Q_{1}^{2}-Q_{2}^{2}\right) \\
& +\frac{2}{243} E\left[Q_{1}\left(Q_{1}^{2}-Q_{2}^{2}\right)\right]^{2}+\frac{4}{81} E^{\prime}\left(Q_{1}^{2}+\frac{1}{3} Q_{2}^{2}\right)^{3}+\cdots .
\end{aligned}
$$

The full expansion up to sixth order in $Q_{1}$ and $Q_{2}$ allows for a description of either a first-order or a second-order $I-N_{b}$ transition, depending on the values of the coefficients $A-$ $E^{\prime}$. 
A simplified expansion with $C>0, E>0$, and $D=E^{\prime}=0$ has been studied by Gramsbergen et al. [8]. de Gennes and Prost [6] discuss a slightly more general expansion with $C>0, E>0, D \neq 0$, and $E^{\prime}=0$. In both cases, a second-order $I-N_{b}$ transition is described for $B=0$. For all other values of $B$, the direct $I-N_{b}$ transition does not exist, but is replaced by $I-N_{u}$ and $N_{u}-N_{b}$ transitions. Clearly, the expansion (17) is not appropriate to describe these successive transitions, as it is based on the symmetry breaking at a direct $I-N_{b}$ transition. Expansion (17) can be seen as an approximation of a more general expansion, the approximation being valid close to a direct $I-N_{b}$ transition.

The $N_{u}-N_{b}$ transition is properly described by an expansion of $g_{L, u}$ in powers of the biaxial tensor order parameter $\mathbf{Q}^{b}$. The tensors that appear in this expansion must have the symmetry of the uniaxial $\left(\mathbf{Q}^{b}=\mathbf{0}\right)$ phase, i.e., these tensors are combinations of $s, \delta_{\alpha \beta}, p \varepsilon_{\alpha \beta \gamma}$, and $N_{\alpha \beta}$. The expansion reads

$$
g_{L, u}=g_{\text {uni }}+A Q_{2}^{2}+C Q_{2}^{4}+E Q_{2}^{6}+\cdots,
$$

where $g_{\text {uni }}$ denotes the Landau free-energy density of the uniaxial phase. This free-energy density $g_{\text {uni }}$ and the coefficients $A, C, E, \ldots$ are functions of $Q_{1}, T$, and $p$.

Although Eq. (18) suffices for a description of the $N_{u^{-}}$ $N_{b}$ transition, one can expand this expression further, either with respect to the isotropic phase or with respect to the uniaxial phase.

In the first case, $g_{L, u}$ is expanded in powers of both $\mathbf{Q}^{u}$ and $\mathbf{Q}^{b}$

$$
\begin{aligned}
g_{L, u}\left(\mathbf{Q}^{u}, \mathbf{Q}^{b}, T, p\right)= & g_{\text {iso }}(T, p)+Z_{\alpha \beta}^{u} Q_{\alpha \beta}^{u}+Z_{\alpha \beta}^{b} Q_{\alpha \beta}^{b} \\
& +A_{\alpha \beta \gamma \delta}^{u, u} Q_{\alpha \beta}^{u} Q_{\gamma \delta}^{u}+A_{\alpha \beta \gamma \delta}^{u, b} Q_{\alpha \beta}^{u} Q_{\gamma \delta}^{b} \\
& +A_{\alpha \beta \gamma \delta}^{b, b} Q_{\alpha \beta}^{b} Q_{\gamma \delta}^{b}+\cdots,
\end{aligned}
$$

where $g_{\text {iso }}$, the Gibbs free-energy density of the isotropic phase, and the tensors $\mathbf{Z}^{u}, \mathbf{Z}^{b}, \ldots$ depend on $T$ and $p$. Using isotropic tensors, we find

$$
\begin{aligned}
g_{L, u}\left(\mathbf{Q}^{u}, \mathbf{Q}^{b}, T, p\right)= & g_{\text {iso }}+A_{1} Q_{1}^{2}+A_{2} Q_{2}^{2}+B_{1} Q_{1}^{3}+B_{2} Q_{1} Q_{2}^{2} \\
& +C_{1} Q_{1}^{4}+C_{2} Q_{1}^{2} Q_{2}^{2}+C_{3} Q_{2}^{4}+\cdots, \quad(20)
\end{aligned}
$$

which is the general expansion for a description of successive $I-N_{u}$ and $N_{u}-N_{b}$ transitions.

In the second case, $g_{L, u}$ is expanded in powers of $\mathbf{Q}^{b}$ and $\delta \mathbf{Q}^{u} \equiv \mathbf{Q}^{u}-\mathbf{Q}^{u, \dagger}$, where $\mathbf{Q}^{u, \dagger}$ is the equilibrium tensor order parameter describing a uniform uniaxial phase at a suitably chosen temperature $T^{\dagger}$ and pressure $p^{\dagger}$. Using an expansion analogous to Eq. (19), but now with uniaxial tensors, we find

$$
\begin{aligned}
g_{L, u}\left(\mathbf{Q}^{u}, \mathbf{Q}^{b}, T, p\right)= & g_{\text {uni }}+Z_{1} \delta Q_{1}+A_{1} \delta Q_{1}{ }^{2}+A_{2} Q_{2}^{2} \\
& +B_{1} \delta Q_{1}{ }^{3}+B_{2} \delta Q_{1} Q_{2}^{2}+C_{1} \delta Q_{1}{ }^{4} \\
& +C_{2} \delta Q_{1}{ }^{2} Q_{2}^{2}+C_{3} Q_{2}^{4}+\cdots
\end{aligned}
$$

It should be remarked that the uniaxial symmetry allows for an additional term linear in $\delta Q_{1}$. However, $Z_{1}$ must be zero at $T=T^{\dagger}$ and $p=p^{\dagger}$ since we have defined $Q_{1}^{\dagger}$ to be the equilibrium value of $Q_{1}$ at this temperature and pressure.

Summarizing, we have demonstrated how an approximate expression for the free-energy density of a nematic phase can be obtained by an expansion with respect to a higher symmetric phase. For uniaxial nematic liquid crystals, this is an expansion with respect to the isotropic phase [Eq. (15)]. For biaxial nematic liquid crystals, three different approximations can be used: a direct expansion with respect to the isotropic phase [Eq. (17)], an indirect expansion with respect to the isotropic phase through an intermediate uniaxial nematic phase [Eq. (20)], and an expansion with respect to the uniaxial nematic phase [Eq. (21)].

\section{B. Nonuniform systems: Elasticity}

For nonuniform systems with a smoothly varying order parameter, the Landau free-energy density $g_{L}$ can be expanded in powers of the spatial derivatives of the order parameter, as in Eq. (8). The terms in that expansion that contain only the spatial derivatives of the directors $(\boldsymbol{l}(\boldsymbol{r}), \boldsymbol{m}(\boldsymbol{r}), \boldsymbol{n}(\boldsymbol{r}))$ describe the orientational elasticity of the system. In the generalized elasticity theory, there are also terms that contain only spatial derivatives of the scalar order parameters $\left(Q_{1}(\boldsymbol{r}), Q_{2}(\boldsymbol{r})\right)$, which describe the order elasticicity, and terms that contain both types of derivatives (mixed terms).

In the following, the general expressions for the generalized elastic free-energy density of a uniaxial nematic liquid crystal and of a biaxial nematic liquid crystal are given, where we distinguish between surface elastic terms and bulk elastic terms, as defined in Sec. IIC. We further derive approximate expressions for the generalized elastic free-energy density by making expansions with respect to higher symmetric phases.

\section{Uniaxial nematic liquid crystals}

The contribution of the elastic free-energy density to the Landau free-energy density $g_{L}$ of the uniaxial nematic phase is obtained by substituting expression (6) for the uniaxial tensor order parameter $\mathbf{Q}^{u}$ in expansion (8) of $g_{L}$. The appearing tensors $\boldsymbol{k}, \boldsymbol{K}$, and $\mathcal{K}$ must have the symmetry of the uniaxial phase. All independent elastic terms are found by forming all possible combinations of the tensors with uniaxial symmetry, i.e., combinations of $s, \delta_{\alpha \beta}, p \varepsilon_{\alpha \beta \gamma}$, and $N_{\alpha \beta}$.

We find eight independent bulk terms and four independent surface terms, which can be classified further as four orientational elastic terms, two order elastic terms, two mixed terms, one antisymmetric surface term, and three symmetric surface terms. In terms of the usual vector notation, the elastic free-energy density

$$
g_{L, \mathrm{el}} \equiv g_{L}-g_{L, u}
$$




$$
\begin{aligned}
g_{L, \mathrm{el}}= & k^{u} \boldsymbol{n} \cdot(\boldsymbol{\nabla} \times \boldsymbol{n})+K_{1}^{u}(\boldsymbol{\nabla} \cdot \boldsymbol{n})^{2}+K_{2}^{u}[\boldsymbol{n} \cdot(\boldsymbol{\nabla} \times \boldsymbol{n})]^{2} \\
& +K_{3}^{u}[\boldsymbol{n}(\boldsymbol{\nabla} \times \boldsymbol{n})]^{2}+K_{4}^{u}\left(\boldsymbol{n} \cdot \boldsymbol{\nabla} Q_{1}\right)^{2}+K_{5}^{u}\left(\boldsymbol{\nabla} Q_{1}\right)^{2} \\
& +K_{6}^{u}[\boldsymbol{n} \times(\boldsymbol{\nabla} \times \boldsymbol{n})] \cdot\left(\boldsymbol{\nabla} Q_{1}\right)+K_{7}^{u}(\boldsymbol{\nabla} \cdot \boldsymbol{n})\left(\boldsymbol{n} \cdot \boldsymbol{\nabla} Q_{1}\right) \\
& +\boldsymbol{\nabla} \cdot\left\{S_{1}^{u}[(\boldsymbol{n} \cdot \boldsymbol{\nabla}) \boldsymbol{n}-\boldsymbol{n}(\boldsymbol{\nabla} \cdot \boldsymbol{n})]\right\}+\boldsymbol{\nabla} \cdot\left\{S_{2}^{u} \boldsymbol{\nabla} Q_{1}\right\} \\
& +\boldsymbol{\nabla} \cdot\left\{S_{3}^{u} \boldsymbol{n}\left(\boldsymbol{n} \cdot \boldsymbol{\nabla} Q_{1}\right)\right\}+\boldsymbol{\nabla} \cdot\left\{S_{4}^{u}[(\boldsymbol{n} \cdot \boldsymbol{\nabla}) \boldsymbol{n}+\boldsymbol{n}(\boldsymbol{\nabla} \cdot \boldsymbol{n})]\right\},
\end{aligned}
$$

where all elastic constants are functions of $Q_{1}(\boldsymbol{r}), T$, and $p$. In most literature on orientational elasticity, the elastic constants $K_{1}^{u}, K_{2}^{u}$, and $K_{3}^{u}$ are referred to as $K_{11} / 2, K_{22} / 2$, and $K_{33} / 2$, respectively. The surface elastic constants $S_{1}^{u}$ and $S_{4}^{u}$ are related to the surface elastic constants $K_{24}$ and $K_{13}$, as defined by Nehring and Saupe [24]. As can be seen from the terms corresponding to $K_{6}^{u}$ and $K_{7}^{u}$, a gradient in the order parameter will induce a combined splay-bend deformation of the director field.

It is important to note that the elastic term $k^{u} \boldsymbol{n} \cdot(\boldsymbol{\nabla} \times \boldsymbol{n})$ only appears in the cholesteric $N_{u}^{*}$ phase since it changes sign under spatial inversion. Remarkably, the property of chirality only introduces an orientational elastic term and no chiral order elastic terms. This can be understood as follows. A chiral order elastic term is proportional to $\boldsymbol{n} \cdot \boldsymbol{\nabla} Q_{1}$. Clearly, such a term is forbidden in case of a nonpolar nematic liquid crystal, i.e., a nematic liquid crystal that is symmetric under the substitution $\boldsymbol{n} \rightarrow-\boldsymbol{n}$.

The elastic free-energy expression known from the literature is obtained as an approximation of the general expression presented here. The approximation is based on an expansion with respect to the isotropic phase (see Sec. III A), i.e., the elastic tensors are expanded as in Eqs. (10). The resulting tensors $\mathbf{k}^{0}, \mathbf{K}^{0}$, and $\mathcal{K}^{0}$ have the symmetry of the isotropic phase.

It appears that the elastic free-energy density in this lowest-order approximation can be written in the form of two independent bulk elastic terms, one antisymmetric surface term, and one symmetric surface term. All terms are "mixed" elastic terms

$$
\begin{aligned}
g_{L, \mathrm{el}}= & K_{1}^{i} \partial_{\alpha} Q_{\beta \gamma}^{u} \partial_{\alpha} Q_{\beta \gamma}^{u}+K_{2}^{i} \partial_{\alpha} Q_{\alpha \gamma}^{u} \partial_{\lambda} Q_{\lambda \gamma}^{u} \\
& +S_{1}^{i}\left\{\partial_{\alpha} Q_{\beta \gamma}^{u} \partial_{\beta} Q_{\alpha \gamma}^{u}-\partial_{\alpha} Q_{\alpha \gamma}^{u} \partial_{\lambda} Q_{\lambda \gamma}^{u}\right\}+S_{2}^{i} \partial_{\alpha} \partial_{\beta} Q_{\alpha \beta}^{u} .
\end{aligned}
$$

Note that, e.g., the $K_{1}^{i}$ term is not simply the lowest-order approximation to the $K_{1}^{u}$ term in Eq. (23). Nevertheless, there are relations between the "isotropic" and the "uniaxial" elastic constants, which can be found by substituting the expression for $\mathbf{Q}^{u}$ into Eq. (24).

As follows from Table I the isotropic approximation entails the results

$$
\begin{gathered}
K_{11}=K_{33}>K_{22}, \\
K_{i i} \propto Q_{1}^{2} .
\end{gathered}
$$

In some cases this may be a reasonable approximation. In general, however, Eqs. (25) will not be valid. In that case the isotropic approximation cannot be expected to give a quan-
TABLE I. Bulk elastic terms for the uniaxial nematic phase. The "uniaxial" elastic constants are functions of the uniaxial order parameter, temperature, and pressure. Near the isotropic phase, the elastic constants can be approximated by the expressions in the last column. The "isotropic" elastic constants $K_{1}^{i}$ and $K_{2}^{i}$ are functions of temperature and pressure only.

\begin{tabular}{lcc}
\hline \hline Invariant & Constant & $I$ \\
\hline $\boldsymbol{n} \cdot(\boldsymbol{\nabla} \times \boldsymbol{n})$ & $k^{u}$ & 0 \\
$(\boldsymbol{\nabla} \cdot \boldsymbol{n})^{2}$ & $K_{1}^{u}$ & $\left(2 K_{1}^{i}+K_{2}^{i}\right) Q_{1}^{2}$ \\
{$[\boldsymbol{n} \cdot(\boldsymbol{\nabla} \times \boldsymbol{n})]^{2}$} & $K_{2}^{u}$ & $2 K_{1}^{i} Q_{1}^{2}$ \\
{$[\boldsymbol{n} \times(\boldsymbol{\nabla} \times \boldsymbol{n})]^{2}$} & $K_{3}^{u}$ & $\left(2 K_{1}^{i}+K_{2}^{i}\right) Q_{1}^{2}$ \\
$\left(\boldsymbol{n} \cdot \boldsymbol{\nabla} Q_{1}\right)^{2}$ & $K_{4}^{u}$ & $\frac{1}{3} K_{2}^{i}$ \\
$\left(\boldsymbol{\nabla} Q_{1}\right)^{2}$ & $K_{5}^{u}$ & $\frac{1}{9}\left(6 K_{1}^{i}+K_{2}^{i}\right)$ \\
{$[\boldsymbol{n} \times(\boldsymbol{\nabla} \times \boldsymbol{n})] \cdot \boldsymbol{\nabla} Q_{1}$} & $K_{6}^{u}$ & $\left(4 K_{1}^{i}+\frac{2}{3} K_{2}^{i}\right) Q_{1}$ \\
$(\boldsymbol{\nabla} \cdot \boldsymbol{n})\left(\boldsymbol{n} \cdot \boldsymbol{\nabla} Q_{1}\right)$ & $K_{7}^{u}$ & $\left(4 K_{1}^{i}+\frac{4}{3} K_{2}^{i}\right) Q_{1}$ \\
\hline \hline
\end{tabular}

titatively correct description and one should resort to the general expression (23) presented here.

\section{Biaxial nematic liquid crystals}

The elastic free-energy density $g_{L, \text { el }}$ of the biaxial nematic phase is obtained by substituting expression (3) for the full tensor order parameter $\mathbf{Q}$ in Eq. (8). The appearing tensors $\boldsymbol{k}, \boldsymbol{K}$, and $\mathcal{K}$ must have the symmetry of the biaxial phase. Thus all independent elastic terms are found by forming all possible combinations of biaxially symmetric tensors, i.e., combinations of $s, p \varepsilon_{\alpha \beta \gamma}, L_{\alpha \beta}, M_{\alpha \beta}$, and $N_{\alpha \beta}$ [the Kronecker $\delta$ can be left out due to the completeness relation (5)].

The elastic free-energy density of a nonchiral (chiral) biaxial nematic liquid crystal is described by 33 (36) bulk terms and 12 (12) surface terms. These are given in the Tables III and IV at the end of this section. In these tables we have used the following notation for the invariants that describe orientational deformations [30]:

$$
D_{i j}=\frac{1}{2} \varepsilon_{j k l} R_{i \alpha} R_{k \beta} \partial_{\alpha} R_{l \beta},
$$

where the matrix elements $R_{i \alpha}$ with $i=1,2,3$ and $\alpha=x, y, z$ define the local frame $(\boldsymbol{l}, \boldsymbol{m}, \boldsymbol{n})$ with respect to the space-fixed frame $\left(\boldsymbol{e}_{x}, \boldsymbol{e}_{y}, \boldsymbol{e}_{z}\right)$,

$$
\begin{gathered}
\boldsymbol{l}=R_{1 \alpha} \boldsymbol{e}_{\alpha}, \\
\boldsymbol{m}=R_{2 \alpha} \boldsymbol{e}_{\alpha}, \\
\boldsymbol{n}=R_{3 \alpha} \boldsymbol{e}_{\alpha} .
\end{gathered}
$$

Explicit (vector) expressions of the invariants $D_{i j}$ can be found in Ref. [30]. The appearing chiral terms are orientational elastic terms; chiral order elasticity is also absent here. Of the other bulk terms, 12 are orientational elastic terms, nine are order elastic terms and the remaining 12 are mixed terms. There are three antisymmetric surface terms and nine symmetric surface terms.

Clearly, the number of independent elastic constants is too large to be experimentally accessible. For that reason, sensible approximations are needed. Instead of postulating ad hoc approximations, we propose three possible approxi- 
mations, all based on symmetry arguments. These approximations are similar in character to the quasi-uniaxial approximation proposed for smectic- $C^{*}$ liquid crystals [34].

The first approximation is the isotropic approximation commonly used in the literature. The approximate elastic free-energy expression is obtained by expanding the Landau free-energy density $g_{L}$ in powers of the full tensor order parameter $\mathbf{Q}$ and its spatial derivatives. The expansion of $g_{L, u}$ is given in Eq. (17), whereas the elastic tensors are expanded according to Eqs. (10). Taking into account only the lowest-order terms, the elastic free-energy density can be approximately described by two bulk elastic terms and two surface terms, as in the uniaxial case:

$$
\begin{aligned}
g_{L, \mathrm{el}}= & K_{1}^{i} \partial_{\alpha} Q_{\beta \gamma} \partial_{\alpha} Q_{\beta \gamma}+K_{2}^{i} \partial_{\alpha} Q_{\alpha \gamma} \partial_{\lambda} Q_{\lambda \gamma} \\
& +S_{1}^{i} \partial_{\alpha}\left(Q_{\beta \gamma} \partial_{\beta} Q_{\alpha \gamma}-Q_{\alpha \gamma} \partial_{\beta} Q_{\beta \gamma}\right)+S_{2}^{i} \partial_{\alpha} \partial_{\beta} Q_{\alpha \beta} .
\end{aligned}
$$

The starting point of the other two approximations is an expansion of the Landau free-energy density in which the uniaxial and the biaxial tensor order parameter are treated as independent order parameters:

$$
\begin{aligned}
& g_{L}\left(\boldsymbol{Q}^{u}, \boldsymbol{Q}^{b}, \boldsymbol{\partial}^{(n)} \boldsymbol{Q}^{u}, \boldsymbol{\partial}^{(m)} \boldsymbol{Q}^{b}, T, p\right) \\
&= g_{L, u}\left(\boldsymbol{Q}^{u}, \boldsymbol{Q}^{b}, T, p\right)+k_{\alpha \beta \gamma}^{u}\left(\boldsymbol{Q}^{u}, \boldsymbol{Q}^{b}, T, p\right) \partial_{\alpha} Q_{\beta \gamma}^{u} \\
&+k_{\alpha \beta \gamma}^{b}\left(\boldsymbol{Q}^{u}, \boldsymbol{Q}^{b}, T, p\right) \partial_{\alpha} Q_{\beta \gamma}^{b} \\
&+K_{\alpha \beta \gamma \lambda \mu \nu}^{u, u}\left(\boldsymbol{Q}^{u}, \boldsymbol{Q}^{b}, T, p\right) \partial_{\alpha} Q_{\beta \gamma}^{u} \partial_{\lambda} Q_{\mu \nu}^{u} \\
&+K_{\alpha \beta \gamma \lambda \mu \nu}^{u, b}\left(\boldsymbol{Q}^{u}, \boldsymbol{Q}^{b}, T, p\right) \partial_{\alpha} Q_{\beta \gamma}^{u} \partial_{\lambda} Q_{\mu \nu}^{b} \\
&+K_{\alpha \beta \gamma \lambda \mu \nu}^{b, b}\left(\boldsymbol{Q}^{u}, \boldsymbol{Q}^{b}, T, p\right) \partial_{\alpha} Q_{\beta \gamma}^{b} \partial_{\lambda} Q_{\mu \nu}^{b} \\
&+\mathcal{K}_{\alpha \beta \gamma \delta}^{u}\left(\boldsymbol{Q}^{u}, \boldsymbol{Q}^{b}, T, p\right) \partial_{\alpha} \partial_{\beta} Q_{\gamma \delta}^{u} \\
&+\mathcal{K}_{\alpha \beta \gamma \delta}^{b}\left(\boldsymbol{Q}^{u}, \boldsymbol{Q}^{b}, T, p\right) \partial_{\alpha} \partial_{\beta} Q_{\gamma \delta}^{b}+\cdots
\end{aligned}
$$

where $g_{L, u}$ and the tensors $\mathbf{k}^{u}, \mathbf{k}^{b}, \ldots$ are defined with respect to the biaxial phase. The general form of the elastic free-energy density of the (chiral) biaxial phase is obtained by substituting all possible combinations of biaxial tensors in the tensors of expansion (29). Obviously the same three chiral bulk elastic terms, 33 bulk elastic terms, and 12 surface terms are obtained as when using Eq. (8) (see Tables III and IV).

The elastic tensors in Eq. (29) can be expanded with respect to the isotropic phase or with respect to the uniaxial phase. The expansion with respect to the isotropic phase will be treated first. The elastic terms are expanded according to, e.g.,

$$
k_{\alpha \beta \gamma}^{u}\left(\boldsymbol{Q}^{u}, \boldsymbol{Q}^{b}, T, p\right)=k_{\alpha \beta \gamma}^{u, 0}(T, p)+\cdots,
$$

where all appearing tensors (like $\mathbf{k}^{u, 0}$ ) have the symmetry of the isotropic phase. Taking into account only the lowest-
TABLE II. Surface terms for the uniaxial nematic phase. The "uniaxial" elastic constants $S^{u}$ are functions of the uniaxial order parameter, temperature, and pressure. Near the isotropic phase, the elastic constants can be approximated by the expressions in the last column. The "isotropic" elastic constants are functions of temperature and pressure only.

\begin{tabular}{lc}
\hline \hline Surface term & $I$ \\
\hline$\partial_{\alpha}\left\{S_{1}^{u}\left(n_{\beta} \partial_{\beta} n_{\alpha}-n_{\alpha} \partial_{\beta} n_{\beta}\right)\right\}$ & $S_{1}^{u} \approx\left(2 K_{1}^{i}+S_{1}^{i}\right) Q_{1}^{2}$ \\
$\partial_{\alpha}\left\{S_{2}^{u} \partial_{\alpha} Q_{1}\right\}$ & $S_{2}^{u} \approx-\frac{1}{3} S_{2}^{i}$ \\
$\partial_{\alpha}\left\{S_{3}^{u} N_{\alpha \beta} \partial_{\beta} Q_{1}\right\}$ & $S_{3}^{u} \approx S_{2}^{i}$ \\
$\partial_{\alpha}\left\{S_{4}^{u}\left(n_{\beta} \partial_{\beta} n_{\alpha}+n_{\alpha} \partial_{\beta} n_{\beta}\right)\right\}$ & $S_{4}^{u} \approx S_{2}^{i} Q_{1}$ \\
\hline
\end{tabular}

order terms, we find that, in this approximation, the elastic free-energy density is described by 6 independent bulk elastic terms and 5 independent surface elastic terms

$$
\begin{aligned}
g_{L, \mathrm{el}}= & K_{1}^{i} \partial_{\alpha} Q_{\beta \gamma}^{u} \partial_{\alpha} Q_{\beta \gamma}^{u}+K_{2}^{i} \partial_{\alpha} Q_{\alpha \gamma}^{u} \partial_{\lambda} Q_{\lambda \gamma}^{u}+K_{3}^{i} \partial_{\alpha} Q_{\beta \gamma}^{u} \partial_{\alpha} Q_{\beta \gamma}^{b} \\
& +K_{4}^{i} \partial_{\alpha} Q_{\alpha \gamma}^{u} \partial_{\lambda} Q_{\lambda \gamma}^{b}+K_{5}^{i} \partial_{\alpha} Q_{\beta \gamma}^{b} \partial_{\alpha} Q_{\beta \gamma}^{b} \\
& +K_{6}^{i} \partial_{\alpha} Q_{\alpha \gamma}^{b} \partial_{\lambda} Q_{\lambda \gamma}^{b}+S_{1}^{i} \partial_{\alpha}\left(Q_{\beta \gamma}^{u} \partial_{\beta} Q_{\alpha \gamma}^{u}-Q_{\alpha \gamma}^{u} \partial_{\beta} Q_{\beta \gamma}^{u}\right) \\
& +S_{2}^{i} \partial_{\alpha} \partial_{\beta} Q_{\alpha \beta}^{u}+S_{3}^{i} \partial_{\alpha} \partial_{\beta} Q_{\alpha \beta}^{b} \\
& +S_{4}^{i} \partial_{\alpha}\left(Q_{\beta \gamma}^{u} \partial_{\beta} Q_{\alpha \gamma}^{b}-Q_{\alpha \gamma}^{u} \partial_{\beta} Q_{\beta \gamma}^{b}\right) \\
& +S_{5}^{i} \partial_{\alpha}\left(Q_{\beta \gamma}^{b} \partial_{\beta} Q_{\alpha \gamma}^{b}-Q_{\alpha \gamma}^{b} \partial_{\beta} Q_{\beta \gamma}^{b}\right),
\end{aligned}
$$

where the isotropic elastic constants $K^{i}$ and $S^{i}$ are functions of $T$ and $p$. This so-called indirect isotropic approximation contains the isotropic approximation (28) in the limiting case

$$
\begin{gathered}
K_{3}^{i}=2 K_{1}^{i}=2 K_{5}^{i}, \\
K_{4}^{i}=2 K_{2}^{i}=2 K_{6}^{i}, \\
S_{2}^{i}=S_{3}^{i}, \\
S_{4}^{i}=2 S_{5}^{i}=2 S_{1}^{i} .
\end{gathered}
$$

Finally, we can also expand Eq. (29) with respect to the uniaxial nematic phase. The expansion of $g_{L, u}$ is given in Eq. (21). The tensors in the elastic terms are expanded according to, e.g.,

$$
k_{\alpha \beta \gamma}^{u}\left(\boldsymbol{Q}^{u}, \boldsymbol{Q}^{b}, T, p\right)=k_{\alpha \beta \gamma}^{u, 0}\left(\boldsymbol{Q}^{u}, T, p\right)+\cdots,
$$

where the appearing tensors (like $\mathbf{k}^{u, 0}$ ) have the symmetry of the uniaxial phase and only the lowest-order terms are taken into account.

Obviously, the elastic terms containing only the spatial derivatives of $\mathbf{Q}^{u}$ (the $\mathbf{k}^{u, 0}, \mathbf{K}^{u, u, 0}$, and $\mathcal{K}^{u, 0}$ terms) give rise to the 8 uniaxial bulk elastic terms and the 4 uniaxial surface terms (see Tables I and II). The remaining terms (the $\mathbf{k}^{b, 0}$, $\mathbf{K}^{u, b, 0}, \mathbf{K}^{b, b, 0}$, and $\mathcal{K}^{b, 0}$ terms) give rise to 5 additional bulk elastic terms, 1 additional antisymmetric surface term, and 2 additional symmetric surface terms; there are no additional chiral terms. Then

$$
g_{L, \mathrm{el}}=g_{L, \mathrm{el}}^{u}+g_{L, \mathrm{el}}^{b},
$$

where $g_{L, \text { el }}^{u}$ is given by Eq. (23) and $g_{L, \text { el }}^{b}$ by 


$$
\begin{aligned}
g_{L, \mathrm{el}}^{b}= & K_{8}^{u}\left\{\left(\boldsymbol{l} \cdot \boldsymbol{\nabla} Q_{2}\right) D_{32}+\left(\boldsymbol{m} \cdot \boldsymbol{\nabla} Q_{2}\right) D_{31}+2 Q_{2}\left(D_{13} D_{31}-D_{23} D_{32}\right)\right\}+K_{9}^{u}\left\{-\left(\boldsymbol{l} \cdot \boldsymbol{\nabla} Q_{1}\right)\left(\boldsymbol{l} \cdot \boldsymbol{\nabla} Q_{2}\right)+\left(\boldsymbol{m} \cdot \boldsymbol{\nabla} Q_{1}\right)\left(\boldsymbol{m} \cdot \boldsymbol{\nabla} Q_{2}\right)\right. \\
& \left.+2 Q_{2}\left(\boldsymbol{l} \cdot \boldsymbol{\nabla} Q_{1}\right) D_{23}+2 Q_{2}\left(\boldsymbol{m} \cdot \boldsymbol{\nabla} Q_{1}\right) D_{13}\right\}+K_{10}^{u}\left\{\left(\boldsymbol{n} \cdot \boldsymbol{\nabla} Q_{2}\right)^{2}+4 Q_{2}^{2} D_{33}^{2}\right\}+K_{11}^{u}\left\{\left(\boldsymbol{l} \cdot \boldsymbol{\nabla} Q_{2}\right)^{2}+\left(\boldsymbol{m} \cdot \boldsymbol{\nabla} Q_{2}\right)^{2}\right. \\
& \left.+4 Q_{2}^{2}\left(D_{13}^{2}+D_{23}^{2}\right)\right\}+K_{12}^{u}\left\{-Q_{2} D_{23}\left(\boldsymbol{l} \cdot \boldsymbol{\nabla} Q_{2}\right)+Q_{2} D_{13}\left(\boldsymbol{m} \cdot \boldsymbol{\nabla} Q_{2}\right)\right\}+\partial_{\alpha}\left\{S_{5}^{u}\left(\delta_{\alpha \gamma} N_{\beta \delta}-\delta_{\beta \gamma} N_{\alpha \delta}\right) \partial_{\beta} Q_{\gamma \delta}^{b}\right\} \\
& +\partial_{\alpha}\left\{S_{6}^{u}\left(\delta_{\alpha \gamma} N_{\beta \delta}+\delta_{\beta \gamma} N_{\alpha \delta}\right) \partial_{\beta} Q_{\gamma \delta}^{b}\right\}+\partial_{\alpha}\left\{S_{7}^{u} \delta_{\alpha \gamma} \delta_{\beta \delta} \partial_{\beta} Q_{\gamma \delta}^{b}\right\} .
\end{aligned}
$$

The 12 uniaxial bulk elastic constants $K^{u}$ and seven uniaxial surface elastic constants $S^{u}$ are functions of $Q_{1}(\boldsymbol{r}), T$, and $p$.

The general expression for the elastic free-energy density of the biaxial nematic phase and the three approximate expressions are given in the Tables III and IV. It should be remarked that some of the terms of higher order in $Q_{2}$ that are neglected in the uniaxial approximation are included in the indirect isotropic approximation. Within this last approximation they cannot be left out, as they may be of the same order of magnitude as terms of the same order in $Q_{1}$.

\section{SURFACE-INDUCED LIQUID-CRYSTAL ALIGNMENT}

In this section the GLGT is used to analyze the relation between the orientation and order of a nematic liquid crystal in the bulk and at the surface. To that end, consider a nematic liquid crystal that fills the infinite half space $z \geqslant 0$, bounded by an aligning substrate at $z=0$. We choose the $x$ direction of the laboratory frame along the direction of the alignment at the surface (see Fig. 1). The $x-y$ plane is a mirror plane here. For that reason the local orientation of the nematic liquid crystal can be expressed as

$$
\begin{gathered}
\boldsymbol{l}(z)=(0,1,0), \\
\boldsymbol{m}(z)=(-\sin \alpha(z), 0, \cos \alpha(z)), \\
\boldsymbol{n}(z)=(\cos \alpha(z), 0, \sin \alpha(z)),
\end{gathered}
$$

where the so-called tilt angle $\alpha(z)$ is defined as the angle between the director field $\boldsymbol{n}(z)$ and the surface plane.

We assume the nematic liquid crystal to be strongly anchored to the substrate, i.e., the boundary conditions at the surface are fixed,

$$
\begin{gathered}
Q_{1}(z=0)=Q_{1, s}, \\
Q_{2}(z=0)=Q_{2, s}, \\
\alpha(z=0)=\alpha_{s},
\end{gathered}
$$

where we have taken the surface order to be biaxial. In the bulk, the nematic liquid crystal is uniform and uniaxial:

$$
\begin{gathered}
Q_{1}(z \rightarrow \infty)=\bar{Q}_{1}, \\
Q_{2}(z \rightarrow \infty)=0, \\
Q_{1}^{\prime}(z \rightarrow \infty)=0, \\
Q_{2}^{\prime}(z \rightarrow \infty)=0,
\end{gathered}
$$

$$
\alpha^{\prime}(z \rightarrow \infty)=0,
$$

where the prime denotes the spatial derivative with respect to the $z$ coordinate.

The GLGT can be used to obtain the tilt angle and the scalar order parameters as a function of $z$, thereby describing the influence of the surface order $\left(Q_{1, s}, Q_{2, s}\right)$ and orientation $\left(\alpha_{s}\right)$ on the orientation in the bulk, given by $\alpha_{p} \equiv \alpha(z \rightarrow \infty)$. The Landau free-energy density is given by

$$
g_{L}=g_{L, u}+g_{L, \mathrm{el}},
$$

where $g_{L, u}$ has the form of the Landau free-energy density of the uniform state and $g_{L, \text { el }}$ denotes the elastic free-energy density. Variational calculus leads to the set of coupled Euler-Lagrange equations

$$
\begin{gathered}
\frac{\partial g_{L}}{\partial Q_{1}}=\frac{d}{d z}\left[\frac{\partial g_{L}}{\partial Q_{1}^{\prime}}\right], \\
\frac{\partial g_{L}}{\partial Q_{2}}=\frac{d}{d z}\left[\frac{\partial g_{L}}{\partial Q_{2}^{\prime}}\right], \\
\frac{\partial g_{L}}{\partial \alpha}=\frac{d}{d z}\left[\frac{\partial g_{L}}{\partial \alpha^{\prime}}\right] .
\end{gathered}
$$

The order parameters $Q_{1}(z)$ and $Q_{2}(z)$ and the tilt angle $\alpha(z)$ must be solved from these Euler-Lagrange equations and from the boundary conditions (37) and (38). The solution of these equations constitutes a formidable problem as $g_{L, u}$ and the elastic constants appearing in the expression for $g_{L, \mathrm{el}}$ are (unknown) functions of $Q_{1}(z)$ and $Q_{2}(z)$. However, an approximate analytical solution is quite possible. The starting point of the approximation is the assumption that the difference between the order near the surface and the order in the bulk is small, i.e., the assumption that $\delta Q_{1}(z)$ $\equiv Q_{1}(z)-\bar{Q}_{1}$ and $Q_{2}(z)$ are small for all $z$. Then the EulerLagrange equations can be expanded in $\delta Q_{1}(z), Q_{2}(z)$, and their spatial derivatives and solved up to first order. To that end expressions for $g_{L, u}$ and $g_{L, \text { el }}$ up to lowest order in $\delta Q_{1}(z)$ and $Q_{2}(z)$ are needed. 
TABLE III. Bulk elastic terms for the biaxial nematic phase. The 36 "biaxial" elastic constants can be approximated by linear combinations of either 2, 6, or 13 elastic constants, depending on the approximation that is used.

\begin{tabular}{|c|c|c|c|c|}
\hline Invariant & Constant & $N_{u}$ & $I$ (indirect) & $I$ (direct) \\
\hline$D_{11}$ & $k_{1}^{b}$ & $k^{u}$ & 0 & 0 \\
\hline$D_{22}$ & $k_{2}^{b}$ & $k^{u}$ & 0 & 0 \\
\hline$D_{33}$ & $k_{3}^{b}$ & 0 & 0 & 0 \\
\hline$D_{11}^{2}$ & $K_{1}^{b}$ & $K_{2}^{u}$ & $2 K_{1}^{i} Q_{1}^{2}+\frac{2}{3} K_{3}^{i} Q_{1} Q_{2}+\frac{2}{9} K_{5}^{i} Q_{2}^{2}$ & $2 K_{1}^{i}\left(Q_{1}+\frac{1}{3} Q_{2}\right)^{2}$ \\
\hline$D_{22}{ }^{2}$ & $K_{2}^{b}$ & $K_{2}^{u}$ & $2 K_{1}^{i} Q_{1}^{2}-\frac{2}{3} K_{3}^{i} Q_{1} Q_{2}+\frac{2}{9} K_{5}^{i} Q_{2}^{2}$ & $2 K_{1}^{i}\left(-Q_{1}+\frac{1}{3} Q_{2}\right)^{2}$ \\
\hline$D_{33}^{2}$ & $K_{3}^{b}$ & $4 K_{10}^{u} Q_{2}^{2}$ & $\frac{8}{9} K_{5}^{i} Q_{2}^{2}$ & $\frac{8}{9} K_{1}^{i} Q_{2}^{2}$ \\
\hline$D_{12}^{2}$ & $K_{4}^{b}$ & $K_{1}^{u}$ & $\begin{array}{c}\left(2 K_{1}^{i}+K_{2}^{i}\right) Q_{1}^{2} \\
-\frac{1}{3}\left(2 K_{3}^{i}+K_{4}^{i}\right) Q_{1} Q_{2} \\
+\frac{1}{9}\left(2 K_{5}^{i}+K_{6}^{i}\right) Q_{2}^{2}\end{array}$ & $\left(2 K_{1}^{i}+K_{2}^{i}\right)\left(-Q_{1}+\frac{1}{3} Q_{2}\right)^{2}$ \\
\hline$D_{23}{ }^{2}$ & $K_{5}^{b}$ & $4 K_{11}^{u} Q_{2}^{2}$ & $\frac{4}{9}\left(2 K_{5}^{i}+K_{6}^{i}\right) Q_{2}^{2}$ & $\frac{4}{9}\left(2 K_{1}^{i}+K_{2}^{i}\right) Q_{2}^{2}$ \\
\hline$D_{31}^{2}$ & $K_{6}^{b}$ & $K_{3}^{u}$ & $\begin{aligned} & \left(2 K_{1}^{i}+K_{2}^{i}\right) Q_{1}^{2} \\
+ & \frac{1}{3}\left(2 K_{3}^{i}+K_{4}^{i}\right) Q_{1} Q_{2} \\
+ & \frac{1}{9}\left(2 K_{5}^{i}+K_{6}^{i}\right) Q_{2}^{2}\end{aligned}$ & $\left(2 K_{1}^{i}+K_{2}^{i}\right)\left(Q_{1}+\frac{1}{3} Q_{2}\right)^{2}$ \\
\hline$D_{21}^{2}$ & $K_{7}^{b}$ & $K_{1}^{u}$ & $\begin{aligned} & \left(2 K_{1}^{i}+K_{2}^{i}\right) Q_{1}^{2} \\
+ & \frac{1}{3}\left(2 K_{3}^{i}+K_{4}^{i}\right) Q_{1} Q_{2} \\
+ & \frac{1}{9}\left(2 K_{5}^{i}+K_{6}^{i}\right) Q_{2}^{2}\end{aligned}$ & $\left(2 K_{1}^{i}+K_{2}^{i}\right)\left(Q_{1}+\frac{1}{3} Q_{2}\right)^{2}$ \\
\hline$D_{32}^{2}$ & $K_{8}^{b}$ & $K_{3}^{u}$ & $\begin{aligned} & \left(2 K_{1}^{i}+K_{2}^{i}\right) Q_{1}^{2} \\
- & \frac{1}{3}\left(2 K_{3}^{i}+K_{4}^{i}\right) Q_{1} Q_{2} \\
+ & \frac{1}{9}\left(2 K_{5}^{i}+K_{6}^{i}\right) Q_{2}^{2}\end{aligned}$ & $\left(2 K_{1}^{i}+K_{2}^{i}\right)\left(-Q_{1}+\frac{1}{3} Q_{2}\right)^{2}$ \\
\hline$D_{13}^{2}$ & $K_{9}^{b}$ & $4 K_{11}^{u} Q_{2}^{2}$ & $\frac{4}{9}\left(2 K_{5}^{i}+K_{6}^{i}\right) Q_{2}^{2}$ & $\frac{4}{9}\left(2 K_{1}^{i}+K_{2}^{i}\right) Q_{2}^{2}$ \\
\hline$D_{12} D_{21}$ & $K_{10}^{b}$ & $2\left(K_{2}^{u}-K_{1}^{u}\right)$ & $-2 K_{2}^{i} Q_{1}^{2}+\frac{2}{9} K_{6}^{i} Q_{2}^{2}$ & $2 K_{2}^{i}\left(-Q_{1}^{2}+\frac{1}{9} Q_{2}^{2}\right)$ \\
\hline$D_{23} D_{32}$ & $K_{11}^{b}$ & $-2 K_{8}^{u} Q_{2}$ & $\frac{2}{3} K_{4}^{i} Q_{1} Q_{2}-\frac{4}{9} K_{6}^{i} Q_{2}^{2}$ & $\frac{4}{3} K_{2}^{i} Q_{2}\left(Q_{1}-\frac{1}{3} Q_{2}\right)$ \\
\hline$D_{31} D_{13}$ & $K_{12}^{b}$ & $2 K_{8}^{u} Q_{2}$ & $-\frac{2}{3} K_{4}^{i} Q_{1} Q_{2}-\frac{4}{9} K_{6}^{i} Q_{2}^{2}$ & $-\frac{4}{3} K_{2}^{i} Q_{2}\left(Q_{1}+\frac{1}{3} Q_{2}\right)$ \\
\hline$\left(\boldsymbol{l} \cdot \boldsymbol{\nabla} Q_{1}\right)^{2}$ & $K_{13}^{b}$ & $K_{5}^{u}$ & $\frac{1}{9}\left(6 K_{1}^{i}+K_{2}^{i}\right)$ & $\frac{1}{9}\left(6 K_{1}^{i}+K_{2}^{i}\right)$ \\
\hline$\left(\boldsymbol{m} \cdot \nabla Q_{1}\right)^{2}$ & $K_{14}^{b}$ & $K_{5}^{u}$ & $\frac{1}{9}\left(6 K_{1}^{i}+K_{2}^{i}\right)$ & $\frac{1}{9}\left(6 K_{1}^{i}+K_{2}^{i}\right)$ \\
\hline$\left(\boldsymbol{n} \cdot \boldsymbol{\nabla} Q_{1}\right)^{2}$ & $K_{15}^{b}$ & $K_{4}^{u}+K_{5}^{u}$ & $\frac{2}{3}\left(K_{1}^{i}+\frac{2}{3} K_{2}^{i}\right)$ & $\frac{2}{3}\left(K_{1}^{i}+\frac{2}{3} K_{2}^{i}\right)$ \\
\hline$\left(\boldsymbol{l} \cdot \boldsymbol{\nabla} Q_{1}\right)\left(\boldsymbol{l} \cdot \boldsymbol{\nabla} Q_{2}\right)$ & $K_{16}^{b}$ & $-K_{9}^{u}$ & $-\frac{1}{9} K_{4}^{i}$ & $-\frac{2}{9} K_{2}^{i}$ \\
\hline$\left(\boldsymbol{m} \cdot \boldsymbol{\nabla} Q_{1}\right)\left(\boldsymbol{m} \cdot \boldsymbol{\nabla} Q_{2}\right)$ & $K_{17}^{b}$ & $K_{9}^{u}$ & $\frac{1}{9} K_{4}^{i}$ & $\frac{2}{9} K_{2}^{i}$ \\
\hline$\left(\boldsymbol{n} \cdot \boldsymbol{\nabla} Q_{1}\right)\left(\boldsymbol{n} \cdot \boldsymbol{\nabla} Q_{2}\right)$ & $K_{18}^{b}$ & 0 & 0 & 0 \\
\hline$\left(\boldsymbol{l} \cdot \boldsymbol{\nabla} Q_{2}\right)^{2}$ & $K_{19}^{b}$ & $K_{11}^{u}$ & $\frac{1}{9}\left(2 K_{5}^{i}+K_{6}^{i}\right)$ & $\frac{1}{9}\left(2 K_{1}^{i}+K_{2}^{i}\right)$ \\
\hline$\left(\boldsymbol{m} \cdot \boldsymbol{\nabla} Q_{2}\right)^{2}$ & $K_{20}^{b}$ & $K_{11}^{u}$ & $\frac{1}{9}\left(2 K_{5}^{i}+K_{6}^{i}\right)$ & $\frac{1}{9}\left(2 K_{1}^{i}+K_{2}^{i}\right)$ \\
\hline$\left(\boldsymbol{n} \cdot \boldsymbol{\nabla} Q_{2}\right)^{2}$ & $K_{21}^{b}$ & $K_{10}^{u}$ & $\frac{2}{9} K_{5}^{i}$ & $\frac{2}{9} K_{1}^{i}$ \\
\hline$\left(\boldsymbol{l} \cdot \nabla Q_{1}\right) D_{32}$ & $K_{22}^{b}$ & $K_{6}^{u}-\partial K_{2}^{u} / \partial Q_{1}$ & $\frac{2}{3} K_{2}^{i} Q_{1}-\frac{1}{9} K_{4}^{i} Q_{2}$ & $\frac{2}{3} K_{2}^{i}\left(Q_{1}-\frac{1}{3} Q_{2}\right)$ \\
\hline$\left(\boldsymbol{l} \cdot \boldsymbol{\nabla} Q_{1}\right) D_{23}$ & $K_{23}^{b}$ & $2 K_{9}^{u} Q_{2}$ & $\frac{2}{9} K_{4}^{i} Q_{2}$ & $\frac{4}{9} K_{2}^{i} Q_{2}$ \\
\hline$\left(\boldsymbol{m} \cdot \boldsymbol{\nabla} Q_{1}\right) D_{31}$ & $K_{24}^{b}$ & $-K_{6}^{u}+\partial K_{2}^{u} / \partial Q_{1}$ & $-\frac{2}{3} K_{2}^{i} Q_{1}-\frac{1}{9} K_{4}^{i} Q_{2}$ & $-\frac{2}{3} K_{2}^{i}\left(Q_{1}+\frac{1}{3} Q_{2}\right)$ \\
\hline$\left(\boldsymbol{m} \cdot \boldsymbol{\nabla} Q_{1}\right) D_{13}$ & $K_{25}^{b}$ & $2 K_{9}^{u} Q_{2}$ & $\frac{2}{9} K_{4}^{i} Q_{2}$ & ${ }_{\frac{4}{9}} K_{2}^{i} Q_{2}$ \\
\hline$\left(\boldsymbol{n} \cdot \nabla Q_{1}\right) D_{12}$ & $K_{26}^{b}$ & $-K_{7}^{u}+\partial K_{2}^{u} / \partial Q_{1}$ & $-\frac{4}{3} K_{2}^{i} Q_{1}+\frac{2}{9} K_{4}^{i} Q_{2}$ & $\frac{4}{3} K_{2}^{i}\left(-Q_{1}+\frac{1}{3} Q_{2}\right)$ \\
\hline$\left(\boldsymbol{n} \cdot \nabla Q_{1}\right) D_{21}$ & $K_{27}^{b}$ & $K_{7}^{u}-\partial K_{2}^{u} / \partial Q_{1}$ & $\frac{4}{3} K_{2}^{i} Q_{1}+\frac{2}{9} K_{4}^{i} Q_{2}$ & $\frac{4}{3} K_{2}^{i}\left(Q_{1}+\frac{1}{3} Q_{2}\right)$ \\
\hline$\left(\boldsymbol{l} \cdot \boldsymbol{\nabla} Q_{2}\right) D_{32}$ & $K_{28}^{b}$ & $K_{8}^{u}$ & $-\frac{1}{3} K_{4}^{i} Q_{1}+\frac{2}{9} K_{6}^{i} Q_{2}$ & $-\frac{2}{3} K_{2}^{i}\left(Q_{1}-\frac{1}{3} Q_{2}\right)$ \\
\hline$\left(\boldsymbol{l} \cdot \boldsymbol{\nabla} Q_{2}\right) D_{23}$ & $K_{29}^{b}$ & $-K_{12}^{u} Q_{2}$ & $-\frac{4}{9} K_{6}^{i} Q_{2}$ & $-\frac{4}{9} K_{2}^{i} Q_{2}$ \\
\hline$\left(\boldsymbol{m} \cdot \nabla Q_{2}\right) D_{31}$ & $K_{30}^{b}$ & $K_{8}^{u}$ & $-\frac{1}{3} K_{4}^{i} Q_{1}-\frac{2}{9} K_{6}^{i} Q_{2}$ & $-\frac{2}{3} K_{2}^{i}\left(Q_{1}+\frac{1}{3} Q_{2}\right)$ \\
\hline$\left(\boldsymbol{m} \cdot \boldsymbol{\nabla} Q_{2}\right) D_{13}$ & $K_{31}^{b}$ & $K_{12}^{u} Q_{2}$ & $\frac{4}{9} K_{6}^{i} Q_{2}$ & $\frac{4}{9} K_{2}^{i} Q_{2}$ \\
\hline$\left(\boldsymbol{n} \cdot \boldsymbol{\nabla} Q_{2}\right) D_{12}$ & $K_{32}^{b}$ & 0 & 0 & 0 \\
\hline$\left(\boldsymbol{n} \cdot \nabla Q_{2}\right) D_{21}$ & $K_{33}^{b}$ & 0 & 0 & 0 \\
\hline
\end{tabular}

According to Eq. (21), the lowest-order expression for $g_{L, u}$ reads

$$
g_{L, u}=g_{\text {bulk }}+\frac{1}{2} A_{1}\left(\delta Q_{1}\right)^{2}+\frac{1}{2} A_{2} Q_{2}^{2}+\cdots
$$

with $g_{\text {bulk }}$ the free-energy density of the uniform bulk and $A_{1}, A_{2}$ positive constants.

The form of the elastic free-energy density $g_{L, \text { el }}$ can be obtained from Table III. Surface terms (Table IV) can be neglected as we have assumed fixed boundary conditions. As the biaxial order parameter is assumed to be small, $g_{L, \text { el }}$ can 
TABLE IV. Surface terms for the (chiral) biaxial nematic phase. The elastic constants $S^{b}$ are functions of $Q_{1}(\mathbf{r}), Q_{2}(\mathbf{r}), T$, and $p$. The 12 biaxial elastic constants can be approximated as discussed in the text.

\begin{tabular}{lccc}
\hline \hline Surface term & $N_{u}$ & $I$ (indirect) & $I$ (direct) \\
\hline$\partial_{\alpha}\left\{S_{1}^{b}\left(n_{\alpha} \partial_{\beta} n_{\beta}-n_{\beta} \partial_{\beta} n_{\alpha}\right)\right\}$ & $K_{2}^{u}-S_{1}^{u}$ & $-S_{1}^{i} Q_{1}^{2}+\frac{1}{9} S_{5}^{i} Q_{2}^{2}$ & $S_{1}^{i}\left(-Q_{1}^{2}+\frac{1}{9} Q_{2}^{2}\right)$ \\
$\partial_{\alpha}\left\{S_{2}^{b}\left(l_{\alpha} \partial_{\beta} l_{\beta}-l_{\beta} \partial_{\beta} l_{\alpha}\right)\right\}$ & $\frac{1}{3} S_{5}^{u} Q_{2}$ & $\frac{1}{3} S_{4}^{i} Q_{1} Q_{2}-\frac{2}{9} S_{5}^{i} Q_{2}^{2}$ & $\frac{2}{3} S_{1}^{i} Q_{2}\left(Q_{1}-\frac{1}{3} Q_{2}\right)$ \\
$\partial_{\alpha}\left\{S_{3}^{b}\left(m_{\alpha} \partial_{\beta} m_{\beta}-m_{\beta} \partial_{\beta} m_{\alpha}\right)\right\}$ & $-\frac{1}{3} S_{5}^{u} Q_{2}$ & $-\frac{1}{3} S_{4}^{i} Q_{1} Q_{2}-\frac{2}{9} S_{5}^{i} Q_{2}^{2}$ & $-\frac{2}{3} S_{1}^{i} Q_{2}\left(Q_{1}+\frac{1}{3} Q_{2}\right)$ \\
$\partial_{\alpha}\left\{S_{4}^{b} N_{\alpha \beta} \partial_{\beta} Q_{1}\right\}$ & $S_{2}^{u}+S_{3}^{u}$ & $\frac{2}{3} S_{2}^{i}$ & $\frac{2}{3} S_{2}^{i}$ \\
$\partial_{\alpha}\left\{S_{5}^{b} N_{\alpha \beta} \partial_{\beta} Q_{2}\right\}$ & 0 & 0 & 0 \\
$\partial_{\alpha}\left\{S_{6}^{b} M_{\alpha \beta} \partial_{\beta} Q_{1}\right\}$ & $S_{2}^{u}$ & $-\frac{1}{3} S_{2}^{i}$ & $-\frac{1}{3} S_{2}^{i}$ \\
$\partial_{\alpha}\left\{S_{7}^{b} M_{\alpha \beta} \partial_{\beta} Q_{2}\right\}$ & $-\frac{1}{3} S_{7}^{u}$ & $-\frac{1}{3} S_{3}^{i}$ & $-\frac{1}{3} S_{2}^{i}$ \\
$\partial_{\alpha}\left\{S_{8}^{b} L_{\alpha \beta} \partial_{\beta} Q_{1}\right\}$ & $S_{2}^{u}$ & $-\frac{1}{3} S_{2}^{i}$ & $-\frac{1}{3} S_{2}^{i}$ \\
$\partial_{\alpha}\left\{S_{9}^{b} L_{\alpha \beta} \partial_{\beta} Q_{2}\right\}$ & $\frac{1}{3} S_{7}^{u}$ & $\frac{1}{3} S_{3}^{i}$ & $\frac{1}{3} S_{2}^{i}$ \\
$\partial_{\alpha}\left\{S_{10}^{b}\left(-m_{\alpha} D_{31}-n_{\alpha} D_{21}\right)\right\}$ & $-S_{4}^{u}-\frac{1}{3}\left(S_{6}^{u}+S_{7}^{u}\right) Q_{2}$ & $-S_{2}^{i} Q_{1}-\frac{1}{3} S_{3}^{i} Q_{2}$ & $-S_{2}^{i}\left(Q_{1}+\frac{1}{3} Q_{2}\right)$ \\
$\partial_{\alpha}\left\{S_{11}^{b}\left(-n_{\alpha} D_{12}-l_{\alpha} D_{32}\right)\right\}$ & $S_{4}^{u}-\frac{1}{3}\left(S_{6}^{u}+S_{7}^{u}\right) Q_{2}$ & $S_{2}^{i} Q_{1}-\frac{1}{3} S_{3}^{i} Q_{2}$ & $S_{2}^{i}\left(Q_{1}-\frac{1}{3} Q_{2}\right)$ \\
$\partial_{\alpha}\left\{S_{12}^{b}\left(-m_{\alpha} D_{13}-l_{\alpha} D_{23}\right)\right\}$ & $\frac{2}{3} S_{7}^{u} Q_{2}$ & $\frac{2}{3} S_{3}^{i} Q_{2}$ & $\frac{2}{3} S_{2}^{i} Q_{2}$ \\
\hline \hline
\end{tabular}

be approximated according to Eqs. (35) and (23). The following expression for $g_{L, e l}$ is found:

$$
\begin{aligned}
g_{L, \mathrm{el}} \approx & \left(K_{1}^{u} \cos ^{2} \alpha+K_{3}^{u} \sin ^{2} \alpha\right) \alpha^{\prime 2}+\left(K_{5}^{u}+K_{4}^{u} \sin ^{2} \alpha\right)\left(\delta Q_{1}^{\prime}\right)^{2} \\
& +K_{9}^{u} \cos ^{2} \alpha \delta Q_{1}^{\prime} Q_{2}^{\prime}+\left(K_{11}^{u} \cos ^{2} \alpha+K_{10}^{u} \sin ^{2} \alpha\right) Q_{2}^{\prime 2} \\
& +\frac{1}{2}\left(K_{7}^{u}-K_{6}^{u}\right) \sin (2 \alpha) \alpha^{\prime} \delta Q_{1}^{\prime}+\frac{1}{2} K_{8}^{u} \sin (2 \alpha) \alpha^{\prime} Q_{2}^{\prime} .
\end{aligned}
$$

The dependence of the uniaxial elastic constants on $\delta Q_{1}$ can be neglected, as $\delta Q_{1}$ is assumed to be small.

Now the Euler-Lagrange equations can be expanded in terms of $\delta Q_{1}(z)$ and $Q_{2}(z)$. The equations of order zero appear to lead to

$$
\alpha_{0}^{\prime \prime}=0
$$

Taking into account boundary conditions (38), the zerothorder tilt angle profile follows as

$$
\alpha_{0}(z)=\alpha_{p}
$$

This is hardly surprising, as the Frank free energy, i.e., the elastic free energy in the case of uniform order parameters, is minimized by a uniform director profile.

The Euler-Lagrange equations of first order are
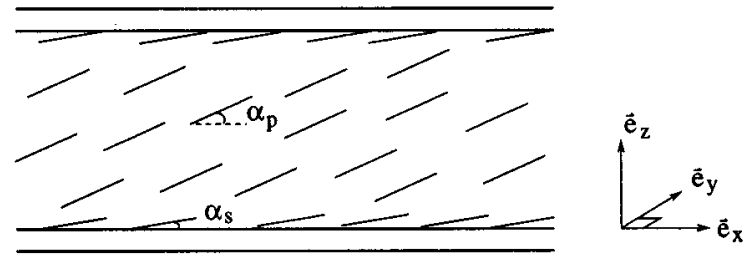

FIG. 1. Nematic cell. The orientation of the director is determined by the tilt angle $\alpha$, the value of which at the surface $\alpha_{s}$ is usually different from the value in the bulk of the nematic liquid crystal. The latter is referred to as the pretilt angle $\alpha_{p}$.

$$
\begin{gathered}
2\left(K_{5}^{u}+K_{4}^{u} \sin ^{2} \alpha_{p}\right) \delta Q_{1}^{\prime \prime}+K_{9}^{u} \cos ^{2} \alpha_{p} Q_{2}^{\prime \prime} \\
+\frac{1}{2}\left(K_{7}^{u}-K_{6}^{u}\right) \sin \left(2 \alpha_{p}\right) \alpha_{1}^{\prime \prime}-A_{1} \delta Q_{1}=0, \\
K_{9}^{u} \cos ^{2} \alpha_{p} \delta Q_{1}^{\prime \prime}+2\left(K_{11}^{u} \cos ^{2} \alpha_{p}+K_{10}^{u} \sin ^{2} \alpha_{p}\right) Q_{2}^{\prime \prime} \\
+\frac{1}{2} K_{8}^{u} \sin \left(2 \alpha_{p}\right) \alpha_{1}^{\prime \prime}-A_{2} Q_{2}=0, \\
2\left(K_{1}^{u} \cos ^{2} \alpha_{p}+K_{3}^{u} \sin ^{2} \alpha_{p}\right) \alpha_{1}^{\prime \prime}+\frac{1}{2}\left(K_{7}^{u}-K_{6}^{u}\right) \sin \left(2 \alpha_{p}\right) \delta Q_{1}^{\prime \prime} \\
+\frac{1}{2} K_{8}^{u} \sin \left(2 \alpha_{p}\right) Q_{2}^{\prime \prime}=0 .
\end{gathered}
$$

The solution to Eq. (45c) can be expressed as

$$
\begin{aligned}
\alpha_{1}(z)= & -\frac{\left(K_{7}^{u}-K_{6}^{u}\right) \sin \left(2 \alpha_{p}\right)}{4\left(K_{1}^{u} \cos ^{2} \alpha_{p}+K_{3}^{u} \sin ^{2} \alpha_{p}\right)} \delta Q_{1}(z) \\
& -\frac{K_{8}^{u} \sin \left(2 \alpha_{p}\right)}{4\left(K_{1}^{u} \cos ^{2} \alpha_{p}+K_{3}^{u} \sin ^{2} \alpha_{p}\right)} Q_{2}(z) .
\end{aligned}
$$

Now Eqs. (45a) and (45b) can be written as

$$
\begin{aligned}
& B_{1} \delta Q_{1}^{\prime \prime}+B_{3} Q_{2}^{\prime \prime}-A_{1} \delta Q_{1}=0, \\
& B_{3} \delta Q_{1}^{\prime \prime}+B_{2} Q_{2}^{\prime \prime}-A_{2} Q_{2}=0,
\end{aligned}
$$

with

$$
\begin{gathered}
B_{1}=2\left(K_{5}^{u}+K_{4}^{u} \sin ^{2} \alpha_{p}\right)-\frac{\left(K_{7}^{u}-K_{6}^{u}\right)^{2} \sin ^{2}\left(2 \alpha_{p}\right)}{8\left(K_{1}^{u} \cos ^{2} \alpha_{p}+K_{3}^{u} \sin ^{2} \alpha_{p}\right)}, \\
B_{2}=2\left(K_{11}^{u} \cos ^{2} \alpha_{p}+K_{10}^{u} \sin ^{2} \alpha_{p}\right)-\frac{K_{8}^{u 2} \sin ^{2}\left(2 \alpha_{p}\right)}{8\left(K_{1}^{u} \cos ^{2} \alpha_{p}+K_{3}^{u} \sin ^{2} \alpha_{p}\right)}, \\
B_{3}=K_{9}^{u} \cos ^{2} \alpha_{p} .
\end{gathered}
$$

It follows that the scalar order parameters vary according to

$$
\delta Q_{1}(z)=C_{1} \exp \left(-a_{+} z\right)+C_{2} \exp \left(-a_{-} z\right)
$$




$$
Q_{2}(z)=C_{3} \exp \left(-a_{+} z\right)+C_{4} \exp \left(-a_{-} z\right),
$$

where the coefficients $a_{ \pm}$are given by

$$
a_{ \pm}=\sqrt{\frac{\left(B_{1} A_{2}+B_{2} A_{1}\right) \pm \sqrt{\left(B_{1} A_{2}-B_{2} A_{1}\right)^{2}+4 A_{1} A_{2} B_{3}^{2}}}{2\left(B_{1} B_{2}-B_{3}^{2}\right)}} .
$$

The lengths $1 / a_{+}$and $1 / a_{-}$are of the order of what is usually called the coherence length. The coefficients $C_{1}-C_{4}$ are given by

$$
\begin{gathered}
C_{1}=\frac{1}{2}\left(1-R_{1}\right) \delta Q_{1, s}-R_{2} Q_{2, s}, \\
C_{2}=\frac{1}{2}\left(1+R_{1}\right) \delta Q_{1, s}+R_{2} Q_{2, s}, \\
C_{3}=\frac{1}{2}\left(1+R_{1}\right) Q_{2, s}-R_{A} R_{2} \delta Q_{1, s}, \\
C_{4}=\frac{1}{2}\left(1-R_{1}\right) Q_{2, s}+R_{A} R_{2} \delta Q_{1, s},
\end{gathered}
$$

with the dimensionless constants

$$
\begin{gathered}
R_{A}=\frac{A_{1}}{A_{2}}, \\
R_{1}=\frac{B_{1} A_{2}-B_{2} A_{1}}{\sqrt{\left(B_{1} A_{2}-B_{2} A_{1}\right)^{2}+4 A_{1} A_{2} B_{3}^{2}}}, \\
R_{2}=\frac{B_{3} A_{2}}{\sqrt{\left(B_{1} A_{2}-B_{2} A_{1}\right)^{2}+4 A_{1} A_{2} B_{3}^{2}}} .
\end{gathered}
$$

Summarizing, the tilt angle profile up to first order in $\delta Q_{1}(z)$ and $Q_{2}(z)$ is

$$
\begin{aligned}
\alpha(z)= & \alpha_{0}(z)+\alpha_{1}(z) \\
= & \alpha_{p}-\frac{\left(K_{7}^{u}-K_{6}^{u}\right) \sin \left(2 \alpha_{p}\right)}{4\left(K_{1}^{u} \cos ^{2} \alpha_{p}+K_{3}^{u} \sin ^{2} \alpha_{p}\right)} \delta Q_{1}(z) \\
& -\frac{K_{8}^{u} \sin \left(2 \alpha_{p}\right)}{4\left(K_{1}^{u} \cos ^{2} \alpha_{p}+K_{3}^{u} \sin ^{2} \alpha_{p}\right)} Q_{2}(z) .
\end{aligned}
$$

The constant $\alpha_{p}$ must be solved from the following equation, which follows from the boundary conditions (37):

$$
\begin{aligned}
\alpha_{s}= & \alpha_{p}-\frac{\left(K_{7}^{u}-K_{6}^{u}\right) \sin \left(2 \alpha_{p}\right)}{4\left(K_{1}^{u} \cos ^{2} \alpha_{p}+K_{3}^{u} \sin ^{2} \alpha_{p}\right)}\left(Q_{1, s}-\bar{Q}_{1}\right) \\
& -\frac{K_{8}^{u} \sin \left(2 \alpha_{p}\right)}{4\left(K_{1}^{u} \cos ^{2} \alpha_{p}+K_{3}^{u} \sin ^{2} \alpha_{p}\right)} Q_{2, s} .
\end{aligned}
$$

In the direct isotropic approximation and in the case of small angles $\alpha_{p}$ and $\alpha_{s}$ this result reduces to

$$
\alpha_{p}=\alpha_{s}\left\{1-\frac{K_{2}^{i}\left[\bar{Q}_{1}-Q_{1, s}+Q_{2, s}\right]}{3\left(2 K_{1}^{i}+K_{2}^{i}\right) \bar{Q}_{1}}\right\}
$$

which is the same result as that obtained in Refs. [16,17].

We conclude that the nonuniformity of the scalar order parameters in a region with a thickness of the order of the coherence length induces a combined splay-bend deformation of the director field in this subsurface region. For a liquid-crystal layer with a thickness much larger than the coherence length, the director profile seems uniformly tilted with a tilt angle $\alpha_{p}$. Thus the interaction of the liquid-crystal with the substrate apparently favors the alignment of the director along a preferential axis that makes an angle $\alpha_{p}$ with the substrate. It follows that $\alpha_{p}$ is equal to the so-called pretilt angle, which is an important parameter for liquid crystal display devices. Knowledge of the relation between the pretilt angle and surface properties such as $\alpha_{s}, Q_{1, s}$, and $Q_{2, s}$, which can be measured using second-harmonic generation [15-17], might be quite useful.

The obtained result has some relevance to the debate on the surface elastic constant $K_{13}$ as well. This elastic constant introduces an artifact in the continuum theory of nematic liquid crystals. A straightforward minimization procedure for the free-energy functional, including the $K_{13}$ term, leads to a discontinuity of the director field at the surface [35]. In order to remedy this artifact of the theory Barbero and co-workers introduced a higher-order elastic constant [36]. This procedure results in a variation of the director tilt in a small layer near the surface. The tilt variation over this subsurface layer appears to be given by

$$
\Delta \alpha \approx \frac{K_{13}}{2 K} \sin \left(2 \alpha_{s}\right),
$$

with $K$ of the order of the Frank elastic constants. This proposal to remedy the $K_{13}$ artifact was subsequently criticized in the literature [37]. A tilt variation close to the surface is also found in the framework of the present GLGT. Moreover, Eq. (54) for the tilt variation over the subsurface layer is quantitatively similar to Eq. (56). For this reason the approach of [36] can be seen as an effective theory for describing the influence of order variations near a surface on the liquid-crystal orientation. As a consequence, $K_{13}$ must then be seen as an effective parameter, related to the order variation near the surface and the elastic constants of the underlying GLGT in the following way:

$$
K_{13}^{\mathrm{eff}} \equiv \frac{1}{2}\left(K_{7}^{u}-K_{6}^{u}\right) \delta Q_{1, s}+\frac{1}{2} K_{8}^{u} Q_{2, s}
$$

In the direct isotropic approximation (see Table I) this reduces to $K_{13}^{\mathrm{eff}} \approx K_{2}^{i} \bar{Q}_{1}\left(\delta Q_{1, s}-Q_{2, s}\right) / 3$. It follows that $K_{13}^{\mathrm{eff}}$ is negative for a disordering surface $\left(\delta Q_{1, s}<0\right)$ and positive for an ordering surface $\left(\delta Q_{1, s}>0\right)$, in the case where biaxiality is neglected. Clearly, $K_{13}^{\text {eff }}$ is not a genuine elastic constant, as it depends on the properties of the substrate with which the liquid crystal is in contact. 


\section{SUMMARY}

The Landau free-energy density and the elastic freeenergy density of nematic and cholesteric liquid crystals with either uniaxial or biaxial symmetry are considered. A tensor analysis is used to find all the terms that are allowed by the symmetries of these liquid-crystalline phases.

The expression for the Landau free-energy density is derived for the isotropic-uniaxial nematic phase transition, the isotropic-biaxial nematic phase transition, and the uniaxial nematic-biaxial nematic phase transition. It appears that a direct isotropic-biaxial nematic transition imposes more severe constraints on the expression for the Landau free-energy density of the biaxial nematic phase than two successive isotropic-uniaxial nematic-biaxial nematic transitions. The expression for the direct isotropic-biaxial nematic transition corresponds to the expression known from the literature. Clearly, this expression is not generally valid.

The same observation holds for the GLGT, which combines Landau theory with elasticity theory. The GLGT known from the literature appears to be an approximation of the more general theory that is investigated in this paper. For biaxial nematic liquid crystals, the well-known approximate GLGT expression, as well as two other approximate expressions, is derived.
Concerning the elastic part of the free-energy density, it is found that uniaxial nematic liquid crystals are described by eight independent bulk elastic terms and four independent surface terms. In the isotropic approximation, the number of bulk terms (surface terms) is reduced to two (two). Biaxial nematic liquid crystals, on the other hand, are described by 36 independent bulk elastic terms and 12 independent surface terms. The number of bulk terms (surface terms) is reduced to 13 (7) in the uniaxial approximation, to six (five) in the indirect isotropic approximation, and to two (two) in the direct isotropic approximation. This last direct isotropic approximation is the approximation corresponding to the GLGT known from the literature

As an illustrative example, the theory has been used to calculate the variation in liquid-crystal orientation in a thin layer near a surface due to variations in the nematic order in this subsurface layer. Such order variations are induced by a substrate imposing order to the liquid crystal differing from the thermodynamically stable order. The resulting tilt variation is quantitatively similar to the variation expected to be caused by the surface elastic constant $K_{13}$. However, the effect described here depends on the type of substrate, whereas the tilt variation due to $K_{13}$ does not.
[1] L. D. Landau, Phys. Z. Sowjetunion 11, 26 (1937); Collected papers of $L$. D. Landau, edited by D. ter Haar (Gordon and Breach, New York, 1965).

[2] L. D. Landau and E. M. Lifshitz, Statistical Physics, 2nd ed. (Pergamon, Oxford, 1969).

[3] P. G. de Gennes, Mol. Cryst. Liq. Cryst. 12, 193 (1971).

[4] R. Alben, Phys. Rev. Lett. 30, 778 (1973).

[5] P. B. Vigman, A. I. Larkin, and V. M. Filev, Zh. Éksp. Teor. Fiz. 68, 1883 (1975) [Sov. Phys. JETP 41, 944 (1976)].

[6] P. G. de Gennes and J. Prost, The Physics of Liquid Crystals, 2nd ed. (Clarendon, Oxford, 1993).

[7] G. Vertogen and W. H. de Jeu, Thermotropic Liquid Crystals, Fundamentals (Springer-Verlag, Berlin, 1988).

[8] E. F. Gramsbergen, L. Longa, and W. H. de Jeu, Phys. Rep. 135, 195 (1986).

[9] E. B. Priestley, P. J. Wojtowicz, and P. Sheng, Introduction to Liquid Crystals (Plenum, New York, 1979).

[10] P. Sheng, Phys. Rev. Lett. 37, 1059 (1976); D. W. Allender, G. L. Henderson, and D. L. Johnson, Phys. Rev. A 24, 1086 (1981); P. Sheng, ibid. 26, 1610 (1982).

[11] T. J. Sluckin and A. Poniewierski, Phys. Rev. Lett. 55, 2907 (1985).

[12] G. Barbero and G. Durand, J. Phys. (France) II 1, 651 (1991).

[13] N. Kothekar, D. W. Allender, and R. M. Hornreich, Phys. Rev. E 49, 2150 (1994); 52, 4541 (1995).

[14] Y. Lansac, F. Fried, and P. Maïssa, Phys. Rev. E 52, 6227 (1995).

[15] M. Barmentlo, R. W. J. Hollering, and N. A. J. M. van Aerle, Phys. Rev. A 46, R4490 (1992).

[16] D. Johannsmann, H. Zhou, P. Sonderkaer, H. Wierenga, B. O. Myrvold, and Y. R. Shen, Phys. Rev. E 48, 1889 (1993).
[17] X. Zhuang, L. Marucci, and Y. R. Shen, Phys. Rev. Lett. 73, 1513 (1994).

[18] N. Schophol and T. J. Sluckin, Phys. Rev. Lett. 59, 2582 (1987).

[19] E. Penzenstadler and H.-R. Trebin, J. Phys. (Paris) 50, 1027 (1989).

[20] C. W. Oseen, Trans. Faraday Soc. 29, 883 (1933).

[21] H. Zocher, Trans. Faraday Soc. 29, 945 (1933).

[22] F. C. Frank, Discuss. Faraday Soc. 25, 19 (1958).

[23] J. L. Ericksen, Arch. Ration. Mech. Anal. 23, 266 (1966).

[24] J. Nehring and A. Saupe, J. Chem. Phys. 54, 337 (1971).

[25] M. Liu, Phys. Rev. A 24, 2720 (1981).

[26] H.-R. Trebin, J. Phys. (Paris) 42, 1573 (1981).

[27] H. Brandt and H. Pleiner, Phys. Rev. A 24, 2777 (1981).

[28] M. Saslow, Phys. Rev. A 25, 3350 (1982).

[29] E. Govers and G. Vertogen, Phys. Rev. A 30, 1998 (1984).

[30] S. Stallinga and G. Vertogen, Phys. Rev. E 49, 1483 (1994).

[31] K. Schiele and S. Trimper, Phys. Status Solidi B 118, 267 (1983); D. W. Berreman and S. Meiboom, Phys. Rev. A 30, 1955 (1984).

[32] P. Tolédano, A. M. Figueiredo Neto, V. Lorman, B. Mettout, and V. Dmitriev, Phys. Rev. E 52, 5040 (1995).

[33] L. Longa and H.-R. Trebin, Phys. Rev. A 42, 3453 (1990).

[34] S. Stallinga and G. Vertogen, Liq. Cryst. 19, 647 (1995).

[35] C. Oldano and G. Barbero, J. Phys. (Paris) Lett. 46, 451 (1985); Phys. Lett. 110A, 213 (1985); G. Barbero and C. Oldano, Nuovo Cimento D 6, 479 (1985).

[36] G. Barbero, N. V. Madhusudana, and C. Oldano, J. Phys. (Paris) 50, 2263 (1989); G. Barbero, A. Sparavigna, and A. Strigazzi, Nuovo Cimento D 12, 1259 (1990).

[37] V. M. Pergamenshchik, Phys. Rev. E 48, 1254 (1993); S. Stallinga and G. Vertogen, ibid. 53, 1692 (1996). 